Revista de Psicología Vol. 39 (2), 2021 (e-ISSN 2223-3733)

\title{
Representaciones sobre la justicia: Un estudio comparativo en escolares colombianos ${ }^{1}$
}

\author{
Mario Fernando Gutiérrez-Romero ${ }^{2}$, Wilson López-López ${ }^{3}$, Alejandra \\ Orjuela-García ${ }^{4} \&$ Andrea Escobar-Altare ${ }^{5}$ \\ Pontificia Universidad Javeriana-Colombia
}

Se analizaron las representaciones sociales de la justicia de estudiantes colombianos inscritos desde segundo grado de básica primaria hasta el último año de escolaridad secundaria, su nivel de estructuración y sus similitudes y diferencias en función del grado y el estrato socioeconómico. Desde una perspectiva estructural, se administró una técnica de asociación de palabras a 950 participantes (481 niñas y 469 niños) con el término inductor justicia. Los resultados mostraron que existen diferencias en la estructura de la representación de la justicia en función del estrato socioeconómico. Todos los estudiantes tienen un ideal de justicia que se contrapone con elementos negativos los cuales, en el caso de los colegios oficiales, están ligados a la violencia y la venganza mientras que en los colegios privados están relacionados con la corrupción. La representación de la justicia de los estudiantes de colegios privados es más compleja pues incluye una mayor cantidad de mecanismos para ejercerla, que aquella de los pertenecientes a colegios oficiales.

Palabras clave: justicia, desarrollo, representación social, niños y niñas, estrato socioeconómico.

1 Este artículo tiene su origen en las investigaciones "Representaciones Sociales Sobre la Justicia" ID SIAP 7871 y "Mecanismos Discursivos Presentes en el Posicionamiento Identitario" ID SIAP 7800 financiadas por la Vicerrectoría de Investigaciones de la Pontificia Universidad Javeriana.

2 Licenciado en Psicología, magíster en Psicología y Lingüística y doctor en Lingüística. Docente en la Pontificia Universidad Javeriana, Colombia. Dirección postal : Carrera 7 No. 40 - 62. Contacto: mariogutierrez@javeriana.edu.co. https://orcid.org/0000-0003-3452-8658

3 Licenciado y doctor en Psicología. Docente en la Pontificia Universidad Javeriana, Colombia. Dirección postal : Carrera 7 No. 40 - 62. Contacto: lopezw@javeriana.edu.co. https://orcid. org/0000-0002-2964-0402

4 Licenciada en Psicología. Consultora en Oportunidad Estratégica, Colombia. Dirección postal: Carrera 7 No. 40 - 62. Contacto: alejandra.orjuela@javeriana.edu.co. https://orcid. org/0000-0001-5950-4922

5 Licenciada en Psicología y magíster en Psicología Clínica. Docente en la Pontificia Universidad Javeriana, Colombia. Dirección postal: Carrera 7 No. 40 - 62. https://orcid. org/0000-0002-7373-297X 


\section{Social representations on justice: A comparative study in Colombian schoolchildren}

Social representations of justice of Colombian students enrolled from second grade of primary school to the last year of secondary schooling were analyzed. the level of structuring of these representations was established and their similarities and differences were identified according to the school grade and socioeconomic level. From a structural perspective, a word association technique was administered to 950 participants ( 481 girls and 469 boys) with the term justice inducer. The results showed that there are differences in the structure of the representation of justice depending on the socioeconomic stratum. All students have an ideal of justice that is opposed by negative elements which, in the case of official schools, are linked to violence and revenge while in private schools they are related to corruption. The representation of justice of students from private schools is more complex since it includes a greater number of mechanisms to exercise it, than that of those belonging to official schools.

Keywords: justice, development, social representation, children, socioeconomic status.

\section{Representaçóes sobre justiça: Um estudo comparativo em escolares colombianos}

Foram analisadas as representaçóes sociais da justiça de estudantes colombianos do segundo ano do ensino fundamental ao último ano do ensino médio, o nível de estrutura e as semelhanças e diferenças de acordo com o grau e o estrato socioeconômico. Do ponto de vista estrutural, uma técnica de associaçáo de palavras foi aplicada a 950 participantes (481 meninas e 469 meninos) com o termo indutor de justiça. Os resultados existentes mostram que existem diferenças na estrutura da representação da justiça, dependendo do nível socioeconômico. Todos os estudantes têm um ideal de justiça que se opóe a elementos negativos que, no caso das escolas oficiais, estão ligados à violência e à vingança, enquanto nas escolas particulares estão relacionados à corrupção. A representação da justiça de estudantes de escolas particulares é mais complexa, pois inclui um maior número de mecanismos para exercê-la do que os pertencentes às escolas oficiais.

Palavras-chave: justiça, desenvolvimento, representação social, crianças, status socioeconômico.

\section{Représentations sur la justice: une étude comparative chez les écoliers colombiens}

Les représentations sociales de la justice des élèves colombiens inscrits de la deuxième année du primaire à la dernière année du secondaire, leur niveau de structuration et leurs similitudes et différences en fonction de la classe et de la strate socio-économique ont été analysés. D'un point de vue structurel, une technique d'association de mots a été administrée à 950 participants (481 filles et 469 garçons) avec le terme justice inductrice. Les résultats ont montré qu'il existe des différences dans la structure de la représentation de la justice selon la strate socio-économique. Tous les élèves ont un idéal de justice qui contraste avec des éléments négatifs qui, dans le cas des écoles officielles, sont liés à la violence et à la vengeance, alors que dans les écoles privées, ils sont liés à la corruption. La représentation de la justice par les élèves des écoles privées est plus complexe car elle comporte un plus grand nombre de mécanismes pour l'exercer que celle de ceux appartenant aux écoles officielles.

Mots clés: justice, développement, représentation sociale, garçons et filles, strate socio-économique 
Este artículo evoca dos campos conceptuales desde la psicología social y la psicología del desarrollo: el concepto de justicia y el de representaciones sociales (en adelante, "RS"). El concepto de justicia ha sido ampliamente abordado en la psicología social por diversos autores. Actualmente se define como el sentido subjetivo del orden moral restaurado y la corrección moral de la respuesta después de una transgresión o una fechoría (Wenzel \& Okimoto, 2014). La justicia es uno de los bloques constitutivos del comportamiento social y se manifiesta en un variado rango de comportamientos y destrezas sociales, permitiendo comprender la perspectiva del otro, interpretar situaciones sociales y responder apropiadamente a diversos contextos.

Rawls (1997), propone la existencia de dos principios que rigen la justicia: el principio de libertad y el principio de igualdad. Según el primer principio, cada persona debe tener un derecho igual al más amplio sistema total de libertades básicas que, a su vez, sea compatible con un sistema similar de libertad para todos. El principio de igualdad, por otro lado, busca generar un mayor beneficio de los menos aventajados y establece que, además de un buen nivel de vida para todos, es necesaria la justa igualdad de oportunidades (Bolívar, 2012; Graybill, et al., 2018; Rawls,1997).

Estos principios deben reflejar acuerdos imparciales ya que solo así pueden ser justos. Así entonces, la forma como se llega a los consensos o principios que organizan la sociedad debe basarse en la imparcialidad, pero ¿cómo se puede llegar a un acuerdo sobre principios imparciales? Según Rawls (1997; citado en Echeverry \& Jaramillo, 2006), esto solo puede lograrse a través de un velo de ignorancia que imposibilite a todos conocer su posición en la sociedad, lo cual obliga a deliberar con imparcialidad. Así, la teoría de Rawls proporciona una forma de construir principios de justicia que no dependan de las posiciones, características e intereses particulares de cada quien, lo cual le apunta a 
una noción de justicia basada en la imparcialidad y la equidad (Rawls, 1997; Sánchez, et al., 2019; Sarmiento-López \& Yáñez-Canal, 2019). Rawls propone entonces una teoría que aborda la justicia ideal.

Sen (1977), por otro lado, se distancia de la idea de una justicia ideal y se concentra en la justicia posible (Campos, 2011; Colmenarejo, 2016; Nussbaum, 2007; Sen, 2010). Es decir, Sen pretende descubrir la forma de plantear la cuestión del mejoramiento de la injusticia, en vez de ofrecer respuestas a las preguntas sobre la justicia perfecta. Para Sen el conocimiento del estado ideal de la justicia es irrelevante. Incluso cuestiona que este pueda ser identificado, pues considera poco probable que pueda alcanzarse un acuerdo unánime sobre cuáles debieran ser los principios de justicia (Migliore, 2011). Así entonces, esta teoría determina un cambio de rumbo en la formulación de la teoría de la justicia pues pretende incluir fórmulas para superar la injusticia, más que definir la justicia perfecta en las sociedades (Colmenarejo, 2013; Shinn, 2015).

Por otro lado, Okimoto et al. (2012) proponen al menos dos formas de comprender el concepto de justicia: una de carácter retributivo -donde la justicia es una afirmación unilateral contra el delincuente- y otra, un punto de vista restaurativo que concibe la justicia como un método para alcanzar un consenso entre las partes afectadas (Ramírez, et al., 2018; Sarmiento-López \& Yáńez-Canal, 2019). La justicia restaurativa es una forma de responder a la conducta delictiva equilibrando las necesidades de la comunidad, las víctimas y los perpetradores (Betancourt, 2019; Dandurand \& Griffiths, 2006). Por otra parte, esta se define desde una perspectiva psicológica como un sistema por el cual los delincuentes son castigados en proporción a la magnitud moral de los daños intencionalmente causados (Barreiro et al., 2014; Barreiro \& Castorina, 2015; Carlsmith \& Darley, 2008).

\section{La interpretación de la justicia en la infancia}

Desde el siglo pasado, Piaget (1932) hacía ya una distinción entre una preferencia por la igualdad (todo el mundo tiene la misma cantidad) versus una preferencia por la equidad (cada parte obtiene lo que 
necesita o merece); Piaget encontró que las preferencias por la igualdad se sobreponen a las preferencias por la equidad desde la edad de nueve años o incluso más. Hasta la década de 1970, la mayoría de las investigaciones sobre razonamiento moral estaban basadas en la teoría de Kohlberg y en los desarrollos teóricos de Rest y los neo-kohlbergianos (1973, 1979; Rest, et al., 1999). Esto implicaba un énfasis en los principios de la justicia y en los conflictos morales en los que las normas, las leyes, las autoridades y las obligaciones formales son fundamentales. Kohlberg construyó su noción de razonamiento moral como una habilidad que cambia paulatinamente hacia lo analítico y racional y que crece cada vez más hacia niveles de razonamiento más sofisticados (Kohlberg, 1969, 1984). Esta visión del juicio moral como una tarea de razonamiento era consistente con una visión clásica del desarrollo cognitivo que lo suponía lineal, secuencial, universal y determinista en cuanto a la forma como ocurre el desarrollo y sus factores explicativos.

Rest (1979) argumentó que la cuestión de la relación entre las etapas del desarrollo moral y diversos dominios de conocimiento no había sido abordada sistemáticamente. Rest concordaba con la afirmación de Kohlberg de que formas cualitativamente diferentes de juicio moral pueden identificarse en los infantes y que ese desarrollo implica el uso creciente de un razonamiento cada vez más sofisticado. No estaba de acuerdo, sin embargo, con la afirmación de Kohlberg de que el desarrollo avanza a través de una secuencia gradual de etapas internamente consistentes. Rest sostuvo que los infantes utilizan simultáneamente diversos tipos de razonamiento apoyándose en el concepto de esquemas, que sería un concepto más maleable que el de las etapas como Kohlberg propuso.

El estudio desde el desarrollo, de la forma como los niños y niñas entre 3 a 10 años de edad comprenden la noción de justicia se ha basado mayoritariamente en la justicia distributiva (Grocke, et al., 2015; House, et al., 2013; Kenward, et al., 2015; Paulus, 2015; Schmidt, et al., 2016; Wittig, et al., 2013). Para Sabbagh y Schmitt (2016) la justicia distributiva se refiere a la justicia percibida de los principios y normas que regulan la distribución de recursos (por ejemplo, esfuerzo, 
necesidad) y a la evaluación de los resultados reales de la distribución en relación con los resultados esperados.

En general, los estudios recientes sobre justicia y desarrollo han mostrado que, aunque los nińos parecen tener una comprensión de la justicia distributiva desde muy temprana edad, los niños alrededor de los tres años tienden a optimizar su propia ganancia sin signos aparentes de sacrificio o generosidad; desde los 5 años los niños tienden a mostrar más equidad en sus intercambios. Su comportamiento no estaría influenciado por factores como las diferencias sociales, demográficas o culturales (Rochat, et al., 2009) pero si por factores como la edad, auto-afectación, la presencia de otro individuo y la cultura (Forsé, et al., 2016). Para Forsé et al. (2016) lo que varía según las culturas es la magnitud del propio interés de los niños.

Las experiencias de orden inmaterial como la desigualdad, la inseguridad o el irrespeto pueden también producir desajustes inter e intrapersonales que afectan negativamente a dichas personas y la forma como comprenden la justicia (Gollwitzer \& van Prooijen, 2016). Según Fasanelli, et al., (2014) el pensamiento cotidiano en materia de justicia es elaborado a partir de nociones que circulan a través de la comunicación interpersonal; esta dinámica transforma estas nociones, conservándolas, transformándolas, pero también organizándolas alrededor de un cierto número de conceptos que difieren notablemente en su significación de individuo a individuo. De allí la posibilidad de formalizar una aproximación posible de las concepciones ordinarias de la justicia fundamentada en la teoría de las representaciones sociales. El objetivo de este estudio es analizar la forma como los niños y niñas representan el concepto de justicia y cómo se organiza dicha representación estructuralmente.

\section{El concepto de representaciones sociales}

Las representaciones sociales son definidas originalmente como una modalidad del conocimiento, cuya función es la elaboración de los comportamientos y la comunicación entre los individuos; la representación sería un corpus organizado de conocimientos gracias al cual 
se haría inteligible la realidad física y social (Moscovici, 1961). Para Jodelet $(1986 ; 2006)$ las representaciones sociales constituyen maneras de interpretar y de pensar la realidad cotidiana, formas de conocimiento sobre el mundo, implicando en la definición las operaciones mentales que los individuos realizan en dichos contextos de cotidianidad. Para Abric (2001a) la representación social es un conjunto de informaciones, de creencias, de opiniones y de actitudes al propósito de un objeto dado que se organiza y estructura de formas concretas en grupos sociales específicos. Según este autor (Abric, 2001b; Lahlou \& Abric, 2011) los elementos constitutivos están jerarquizados, manteniendo entre una relación que determina su significación y el lugar que ocupan dentro del sistema representacional.

El conjunto de estudios sobre justicia y representaciones sociales sigue siendo escaso, particularmente cuando se exploran las comprensiones sociales en estudiantes. Un estudio sobre representaciones sociales sobre la justicia en estudiantes ha mostrado que los niños de básica primaria no tendrían aparentemente una estructura sólida en el núcleo central de la representación social sobre los derechos humanos pues las respuestas de los niños varían constantemente y su reacción es diferente de una situación a otra (Flores \& Jiménez, 2015). Adicionalmente, otros estudios muestran que para los estudiantes universitarios de Chile la justicia no es una palabra definitoria para comprender la relación entre su Estado y sus comunidades indígenas como sí lo es la palabra "injusticia” (Denegri, et al., 2015) y que el núcleo central de la representación social de la justicia expresa un sentido retributivo en estudiantes universitarios argentinos ya que se le vincula con la búsqueda de la verdad y de la igualdad mediante el juez y la ley (Barreiro \& Castorina, 2015).

\section{Contexto de la investigación}

El presente artículo busca responder ¿cómo se estructuran y se transforman las representaciones sociales sobre justicia en los niños y niñas de 3 a 18 años de edad en función del contexto social que viven- 
cian? Para esto, se propone analizar las representaciones sociales de la justicia de estudiantes inscritos desde segundo grado de básica primaria hasta el último año de escolaridad secundaria, establecer el nivel de estructuración de dichas representaciones conforme avanza la edad e identificar sus similitudes y diferencias en función del estrato socioeconómico; Discutir este tema cobra especial importancia en el contexto colombiano, en el cual los niños, niñas y adolescentes no son ajenos a la idea de justicia. En este país es común que los infantes y adolescentes se vean confrontados a situaciones de violencia e injusticia y, debido a esto, a la pérdida de expectativas frente al concepto ideal de justicia.

Colombia ha vivenciado una situación de extrema violencia política desde hace más de sesenta años, durante los cuales se han comprometido diversas dimensiones y dinámicas sociales como lo son la política, la económica, la jurídica, la religiosa, la cultural, entre otras (Castrillón-Guerrero et al., 2018), haciendo del análisis de la dimensión psicosocial de la violencia una necesidad primordial para alcanzar la construcción de la paz (López-López et al., 2019). Esto ha ejercido una clara influencia sobre el comportamiento social y político de los colombianos (Borja, et al., 2009) lo cual, a su vez, ha generado enmarques emocionales y cognitivos relacionados con el miedo, la venganza y la consolidación de prácticas culturales violentas y ha influido en procesos sociopsicológicos como la memoria, la verdad, el perdón, la empatía, la confianza y la justicia (López- López, 2016a; López-López, 2016b).

Además de la violencia sociopolítica, muchos de los infantes y adolescentes colombianos han vivenciado otro tipo de violencias que también influyen en la construcción de sus enmarques emocionales y cognitivos y en sus procesos sociopsicológicos. Entre 2011 y 2017, 259.843 infantes y adolescentes ingresaron al Proceso de Restablecimiento de Derechos por tener sus derechos amenazados, inobservados o vulnerados, bien sea por maltrato físico, psicológico o negligencia (Observatorio del Bienestar de la Niñez y el Instituto Colombiano de Bienestar Familiar, 2018). Solo en el 2018 se conocieron 10.794 casos de violencia intrafamiliar y 22.794 casos de delitos sexuales en pobla- 
ción de niños, niñas y adolescentes (Instituto Nacional de Medicina Legal y Ciencias Forenses, 2019). Los infantes colombianos crecen en este contexto violento el cual influye en su interpretación de la realidad y, como se mencionó anteriormente, en procesos sociopsicológicos como la justicia. Es a la luz de esto que preguntarse por las representaciones sociales sobre justicia en los niños y niñas colombianos cobra tanto sentido.

\section{Método}

\section{Participantes}

La selección de la muestra fue no probabilística y por conveniencia. Participaron 950 estudiantes, de dos aulas de clase por cada grado escolar y colegio, desde segundo grado hasta undécimo grado ( 481 mujeres y 469 hombres; Min= 4 años Max= 18 años). Ninguno de los participantes presentaba una evidente patología grave del desarrollo o de salud, ni estaba reportado como estudiante en bajo rendimiento. Estos criterios de exclusión de las participantes fueron verificados a partir del reporte hecho por los docentes de cada curso. Los niños que aceptaron participar voluntariamente firmaron un asentimiento informado y sus respectivos tutores legales firmaron un consentimiento informado en consonancia con lo solicitado en la Resolución 8430 de 1993 del Ministerio de Salud de Colombia por la cual se establecen las normas científicas, técnicas y administrativas para la investigación en salud. Los dos proyectos en los cuales se origina este artículo fueron revisados éticamente y aprobados por profesionales del Comité de Investigación y Ética de la Facultad de Psicología de la Pontificia Universidad Javeriana (Bogotá). La muestra se define en la tabla 1. 


\section{Tabla 1}

La composición de la muestra en cuanto a sexo, nivel educativo y estrato socioeconómico

\begin{tabular}{lllc}
\hline \multicolumn{1}{c}{ Variable } & \multicolumn{1}{c}{ Nivel } & $N$ & Proporción \\
\hline \multirow{2}{*}{ Sexo } & Femenino & 481 & $50,63 \%$ \\
\multirow{3}{*}{ Nivel educativo } & Masculino & 469 & $49,37 \%$ \\
& Primaria & 496 & $52,21 \%$ \\
\multirow{2}{*}{ Estrato } & Secundaria & 454 & $47,79 \%$ \\
& Alto & 448 & $47,16 \%$ \\
& Bajo & 502 & $52,84 \%$ \\
\hline
\end{tabular}

Fuente: Elaboración propia

\section{Medición}

Se llevó a cabo un estudio ex post facto transversal el cual implica el uso de encuestas o escalas con el objetivo de describir poblaciones en un único momento temporal, eligiendo el investigador a los participantes por poseer una característica que define la naturaleza de los resultados (Montero \& León, 2005). Participaron dos instituciones educativas de la misma localidad de la ciudad de Bogotá, de dos estratos socioeconómicos diferentes según la escala de las empresas públicas de Bogotá (en una escala de uno a seis siendo el uno el más bajo en recursos económicos y el seis el más alto): Un colegio oficial cuyos estudiantes son generalmente de estrato uno y un colegio privado bilingüe cuyos estudiantes son de estrato seis.

\section{Procedimiento}

Los niños fueron encuestados en sus respectivas aulas de clase; después de un acercamiento inicial para explicarles la naturaleza del trabajo a realizar se distribuyeron los cuadernillos los cuales fueron contestados individualmente por cada estudiante. Los investigadores utilizaron la técnica de asociación de palabras con la siguiente consigna "dime cinco palabras que te vienen a la mente cuando piensas en la 
palabra justicia”. Esto en consonancia con la propuesta estructural de Abric (2001b; Lahlou \& Abric, 2011) para indagar las RS pues pone de manifiesto tanto su campo semántico específico como también su estructura jerárquica (Bruno \& Barreiro, 2014).

\section{Análisis de Datos}

Según esta perspectiva estructural las RS (Abric, 2001b; Lahlou \& Abric, 2011) se encuentran conformadas por un núcleo central y un conjunto de elementos periféricos. El núcleo central está constituido por uno o varios elementos que en la estructura de la representación ocupan una posición privilegiada ya que determina la significación y la organización de la representación en función de los nexos inductivos que generan con los elementos de la zona periférica. El núcleo central significa los otros elementos de la representación y determina la naturaleza de los lazos que unen, entre ellos, a los elementos de la representación. Tiene una determinación social y está relacionado con condiciones históricas, sociológicas e ideológicas; así, está directamente asociado a los valores y las normas. Es la base común propiamente social y colectiva que define la homogeneidad del grupo.

La zona de la periferia contiene elementos que están definidos por su relación con el núcleo central: su presencia, ponderación, su valor y función dependen de este. Los elementos en la zona periférica constituyen lo esencial de la representación en su aspecto más cotidiano, concreto y accesible para cada individuo. Abarcan informaciones retenidas, seleccionadas o interpretadas por las personas, sus juicios sobre el objeto y el entorno, sus estereotipos y creencias. Resultan del anclaje de la representación con la realidad y la hacen comprensible para todos. Asociada a características individuales y al contexto inmediato y contingente de los individuos, este permite una integración de los elementos del núcleo central a las experiencias cotidianas de las personas.

Los análisis fueron realizados con el software IRAMUTEQ, que permite realizar un análisis multidimensional de textos y cuestionarios analizando las unidades lexicales específicas y clasificándolas jerárquicamente por medio de análisis de clústeres, en base a las semejanzas 
y diferencias en el uso de las formas que se da entre los diferentes componentes de dicha población (Molina, 2017). El corpus estuvo constituido por 2520 palabras (506 formas diferentes). Este fue inicialmente "lematizado" por los investigadores, es decir, se trabajó sobre las diversas formas gramaticales presentes para lograr finalmente un corpus exento de sinónimos y con una entrada lexical invariablemente en singular y en género masculino.

\section{Resultados}

El corpus de palabras asociadas por los estudiantes de básica primaria de colegio oficial estuvo constituido por 661 palabras (108 formas diferentes, 80 formas aparecieron solo una vez) y el de los estudiantes de secundaria estuvo constituido por 578 palabras (153 formas diferentes, 91 formas aparecieron solo una vez). El corpus de palabras asociadas por los estudiantes de básica primaria de colegio privado estuvo constituido por 444 palabras (83 formas diferentes, 30 formas aparecieron solo una vez) y el de los estudiantes de secundaria estuvo constituido por 837 palabras (162 formas diferentes, 78 formas aparecieron solo una vez).

A partir de la distribución de las asociaciones se determinó cinco como frecuencia mínima para cada término lo cual implicó analizar el $76,6 \%$ del corpus total de asociaciones. La estructura de la representación social sobre la justicia en niños y niñas de básica primaria de colegio oficial de estrato socioeconómico bajo se observa en la Tabla 2 . 


\section{Tabla 2}

Estructura de la representación social de la justicia; infantes de básica primaria de colegio oficial $(N=661)$

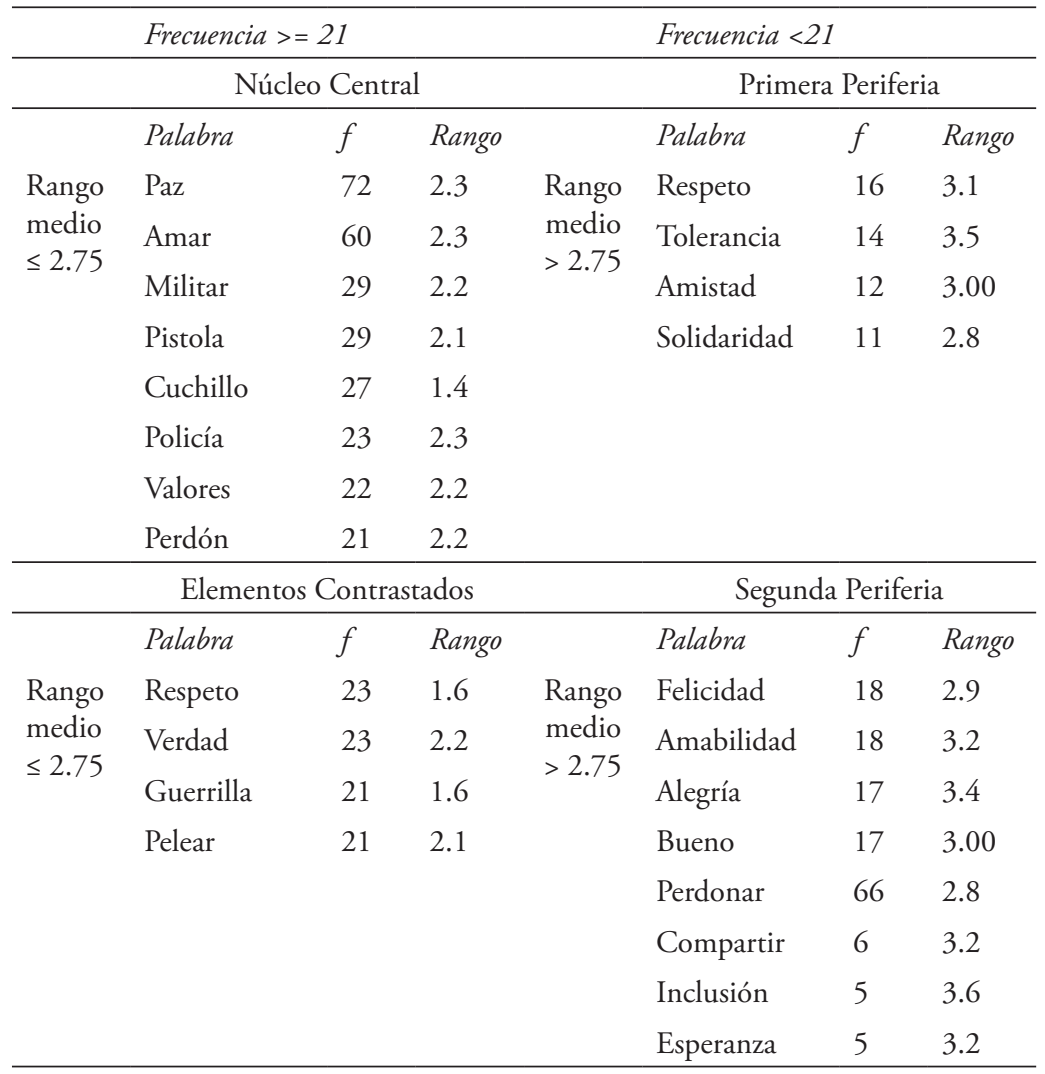

Fuente: Elaboración propia

La estructura de la representación social sobre la justicia en adolescentes de secundaria de colegio oficial de estrato socioeconómico bajo se observa en la Tabla 3. 


\section{Tabla 3}

Estructura de la representación social de la justicia; adolescentes de secundaria de colegio oficial $(N=578)$

\begin{tabular}{|c|c|c|c|c|c|c|c|}
\hline & \multicolumn{4}{|c|}{ Frecuencia $>=22$} & \multicolumn{3}{|l|}{ Frecuencia $<22$} \\
\hline & \multicolumn{4}{|c|}{ Núcleo Central } & \multicolumn{3}{|c|}{ Primera Periferia } \\
\hline \multirow{10}{*}{$\begin{array}{l}\text { Rango } \\
\text { medio } \\
\leq 2.4\end{array}$} & Palabra & $f$ & Rango & \multirow{10}{*}{$\begin{array}{c}\text { Rango } \\
\text { medio } \\
>2.4\end{array}$} & Palabra & $f$ & Rango \\
\hline & Igualdad & 65 & 2.1 & & Respeto & 21 & 2.5 \\
\hline & Verdad & 34 & 1.8 & & Honestidad & 20 & 2.6 \\
\hline & Libertad & 26 & 2.4 & & $\mathrm{Paz}$ & 20 & 2.6 \\
\hline & Derechos & 25 & 2.2 & & Amar & 18 & 2.8 \\
\hline & Equidad & 24 & 2.3 & & Responsabilidad & 17 & 2.6 \\
\hline & Cárcel & 25 & 2.4 & & Tolerancia & 11 & 3.6 \\
\hline & \multirow[t]{3}{*}{ Venganza } & \multirow[t]{3}{*}{22} & \multirow[t]{3}{*}{1.8} & & Deberes & 9 & 2.7 \\
\hline & & & & & Poder & 9 & 2.7 \\
\hline & & & & & Solidaridad & 9 & 3.1 \\
\hline & \multicolumn{3}{|c|}{ Elementos Contrastados } & & \multicolumn{3}{|c|}{ Segunda Periferia } \\
\hline \multirow{7}{*}{$\begin{array}{l}\text { Rango } \\
\text { medio } \\
\leq 2.4\end{array}$} & Palabra & $f$ & Rango & \multirow{7}{*}{$\begin{array}{c}\text { Rango } \\
\text { medio } \\
>2.4\end{array}$} & Palabra & $f$ & Rango \\
\hline & Lealtad & 28 & 2.4 & & Valor & 7 & 2.9 \\
\hline & Ley & 26 & 2.3 & & Sinceridad & 7 & 2.9 \\
\hline & Corrupción & 26 & 2.3 & & Justicia & 7 & 2.9 \\
\hline & Policía & 26 & 2.3 & & Bienestar & 6 & 2.8 \\
\hline & Juez & 25 & 1.8 & & Comprensión & 5 & 3.2 \\
\hline & & & & & Cumplimiento & 5 & 2.8 \\
\hline
\end{tabular}

Fuente: Elaboración propia

La estructura de la representación social sobre la justicia en niños y niñas de básica primaria de colegio privado de estrato socioeconómico alto se observa en la Tabla 4. 
Representaciones sobre la justicia: estudio comparativo en escolares colombianos / Gutiérrez-Romero et al.

\section{Tabla 4}

Estructura de la representación social de la justicia; infantes de básica primaria de colegio privado $(N=444)$

\begin{tabular}{|c|c|c|c|c|c|c|c|}
\hline & \multicolumn{4}{|c|}{ Frecuencia $>=14$} & \multicolumn{3}{|l|}{ Frecuencia $<14$} \\
\hline & \multicolumn{4}{|c|}{ Núcleo Central } & \multicolumn{3}{|c|}{ Primera Periferia } \\
\hline \multirow{10}{*}{$\begin{array}{l}\text { Rango } \\
\text { medio } \\
\leq 2.22\end{array}$} & Palabra & $f$ & Rango & \multirow{10}{*}{$\begin{array}{l}\text { Rango } \\
\text { medio } \\
>2.22\end{array}$} & Palabra & $f$ & Rango \\
\hline & Libertad & 34 & 1.7 & & Anticorrupción & 13 & 2.5 \\
\hline & $\mathrm{Paz}$ & 31 & 2.2 & & Igualdad & 13 & \\
\hline & Respeto & 23 & 2.0 & & Cárcel & 12 & 2.8 \\
\hline & Derechos & 21 & 2.2 & & Policía & 12 & 2.8 \\
\hline & Venganza & 18 & 1.6 & & Honestidad & 11 & 2.7 \\
\hline & Corrupción & 14 & 1.8 & & Juez & 9 & 2.8 \\
\hline & Democracia & 14 & 2.1 & & Solución & 9 & 3.6 \\
\hline & \multirow[t]{2}{*}{ Política } & \multirow[t]{2}{*}{14} & \multirow[t]{2}{*}{2.2} & & Correcto & 8 & 2.6 \\
\hline & & & & & Abogado & 8 & 3.4 \\
\hline & \multicolumn{3}{|c|}{ Elementos Contrastados } & & \multicolumn{3}{|c|}{ Segunda Periferia } \\
\hline \multirow{7}{*}{$\begin{array}{l}\text { Rango } \\
\text { medio } \\
\leq 2.22\end{array}$} & Palabra & $f$ & Rango & \multirow{7}{*}{$\begin{array}{l}\text { Rango } \\
\text { medio } \\
>2.22\end{array}$} & Palabra & $f$ & Rango \\
\hline & Verdad & 17 & 1.6 & & Amabilidad & 7 & 3.4 \\
\hline & Poder & 16 & 2.2 & & Bienestar & 6 & 2.8 \\
\hline & Liderazgo & 15 & 1.6 & & Protección & 6 & 2.8 \\
\hline & & & & & Ley & 6 & 2.7 \\
\hline & & & & & Servicio & 5 & 3.6 \\
\hline & & & & & Cumplimiento & 5 & 3.2 \\
\hline
\end{tabular}

Fuente: Elaboración propia

La estructura de la representación social sobre la justicia en adolescentes de secundaria de colegio privado de estrato socioeconómico alto se observa en la Tabla 5. 


\section{Tabla 5}

Estructura de la representación social de la justicia; adolescentes de secundaria de colegio privado $(N=837)$

\begin{tabular}{|c|c|c|c|c|c|c|c|}
\hline & \multicolumn{4}{|l|}{ Frecuencia $>=23$} & \multicolumn{3}{|l|}{ Frecuencia $<23$} \\
\hline & \multicolumn{4}{|c|}{ Núcleo Central } & \multicolumn{3}{|c|}{ Primera Periferia } \\
\hline \multirow{15}{*}{$\begin{array}{l}\text { Rango } \\
\text { medio } \\
\leq 2.7\end{array}$} & Palabra & $f$ & Rango & \multirow{15}{*}{$\begin{array}{c}\text { Rango } \\
\text { medio } \\
>2.7\end{array}$} & Palabra & $f$ & Rango \\
\hline & Igualdad & 66 & 1.9 & & Paz & 22 & 3.5 \\
\hline & Ley & 52 & 2.1 & & Derechos & 21 & 3.6 \\
\hline & Equidad & 51 & 2.0 & & Cárcel & 20 & 3.7 \\
\hline & Corrupción & 38 & 2.3 & & Verdad & 21 & 2.8 \\
\hline & Honestidad & 32 & 2.3 & & Política & 20 & 2.8 \\
\hline & Libertad & 23 & 2.0 & & Juez & 15 & 3.0 \\
\hline & Poder & 28 & 2.1 & & Bienestar & 14 & 3.6 \\
\hline & Política & 23 & 2.0 & & Respeto & 13 & 3.9 \\
\hline & & & & & Corte & 12 & 3.5 \\
\hline & & & & & Castigo & 10 & 3.0 \\
\hline & & & & & Ética & 9 & 3.1 \\
\hline & & & & & Juicio & 9 & 3.0 \\
\hline & & & & & Abogado & 9 & 2.8 \\
\hline & & & & & Moral & 9 & 2.8 \\
\hline \multicolumn{5}{|c|}{ Elementos Contrastados } & \multicolumn{3}{|c|}{ Segunda Periferia } \\
\hline \multirow{7}{*}{$\begin{array}{l}\text { Rango } \\
\text { medio } \\
\leq 2.7\end{array}$} & Palabra & $f$ & Rango & \multirow{7}{*}{$\begin{array}{c}\text { Rango } \\
\text { medio } \\
>2.7\end{array}$} & Palabra & $f$ & Rango \\
\hline & Democracia & 28 & 2.4 & & Valores & 7 & 3.1 \\
\hline & Equilibrio & 26 & 2.0 & & Fiscal & 6 & 2.8 \\
\hline & Orden & 26 & 2.0 & & Pena & 6 & 2.8 \\
\hline & Corrupto & 23 & 2.0 & & Transparencia & 6 & 2.8 \\
\hline & Gobierno & 26 & 2.0 & & Juzgado & 5 & 3.4 \\
\hline & Anticorrupción & 24 & 2.2 & & & & \\
\hline
\end{tabular}

Fuente: Elaboración propia 


\section{Discusión}

Como se mencionó anteriormente, la perspectiva estructural de las RS desarrollada por la escuela de Aix-en-Provence plantea que las RS se componen de un núcleo central y de elementos periféricos: el núcleo central incluye los elementos más consensuados y estables constituyendo así la identidad de las RS, mientras que la periferia representa la forma como se vive la RS en la cotidianidad. Como puede verse en la tabla 2, las palabras que componen el núcleo central de la RS de los infantes de básica primaria de colegio oficial que participaron en este estudio son paz, amar, militar, pistola, cuchillo, policía, valores y perdón. En la primera periferia, en cambio, se encuentran las palabras respeto, tolerancia, amistad y solidaridad.

Se evidencia así que la base común de la RS de la justicia de niños y niñas de básica primaria de colegio oficial de estrato socioeconómico bajo incluye valores como el amor, la paz y el perdón, junto con elementos que van en una dirección contraria a estos valores, es decir, que hacen referencia a la violencia, el castigo y la represión (lo cual se evidencia en la presencia de palabras como pistola, cuchillo, policía y militar). Pareciera entonces que este núcleo central - el cual refleja las condiciones históricas, sociológicas e ideológicas a partir de las cuales estos niños han construido la RS de la justicia - manifiesta una contradicción. Estos infantes construyen su noción de justicia aludiendo al amor, la paz y el perdón y a su vez, a la violencia. Esta discordancia podría ser explicada a través de lo dicho por Flores y Jiménez (2015), quienes plantean que los nińos de básica primaria aun no tienen una estructura sólida en el núcleo central de las $\mathrm{RS}$ ya que su respuesta varía constantemente de acuerdo con las situaciones particulares que se les presenten. Lo anterior de la mano con el hecho de que los infantes suelen utilizar simultáneamente diversos tipos de razonamiento o esquemas para conceptualizar este tipo de conceptos (Rest, 1997).

Seguidamente la primera periferia de la RS de este grupo de participantes se conforma a través de las palabras respeto, tolerancia, amistady solidaridad. Esto evidencia que los nińos y niñas de colegio oficial viven 
la justicia en su cotidianidad y la hacen tangible desde estos valores. Así, puede decirse que la forma como los infantes experimentan la justicia en su día a día es más cercana a una justicia ideal (Rawls, 1997), que la forma como la piensan y la conceptualizan.

Puede decirse entonces que, en el caso de los niños y niñas de básica primaria de colegio oficial, el centro de la RS de la justicia se idealiza en lo cotidiano. Esto es contrario a lo que ocurre con otros grupos poblacionales como los estudiantes universitarios (Orjuela, 2018) ya que estos piensan la justicia desde lo ideal (reflejado en el núcleo central), lo cual se des-idealiza en lo cotidiano debido a los procesos de administración de justicia y a las instituciones que los implementan. Lo anterior puede atribuirse a la corta edad de los estudiantes de básica primaria, lo cual limita su conocimiento de las instituciones de administración de justicia.

Lo evidenciado con respecto a los niños y niñas de básica primaria de colegio oficial es muy similar a lo que ocurre con los adolescentes en este tipo de colegios. Como lo muestra la Tabla 3, el núcleo central de la RS de adolescentes de secundaria de colegio oficial está compuesto por las palabras igualdad, verdad, libertad, derechos, equidad, cárcel y venganza. La primera periferia, en cambio, se compone de las palabras respeto, honestidad, paz, amar, responsabilidad, tolerancia, deberes, poder y solidaridad. Puede verse que, al igual que en los estudiantes de básica primaria, en el núcleo central se encuentran palabras que aluden a valores positivos y a un tipo de justicia de tipo restaurativo (Okimoto et al., 2012; Dandurand \& Griffiths, 2006; Ramírez, et al., 2018; Sarmiento - López \& Yánez-Canal, 2019; Betancourt, 2019), pero también palabras que hacen referencia a lo retributivo como lo son cárcel y venganza (Barreiro et al., 2014; Barreiro \& Castorina, 2015;Carlsmith \& Darley, 2008; Okimoto et al., 2012).

Asimismo, se evidencia que la primera periferia está compuesta por palabras que denotan valores a partir de los cuales se construye un ideal de justicia. Es decir, allí se muestran los valores desde los cuales se vive la justicia en la cotidianidad. Es notorio que los estudiantes de colegios oficiales (tanto de básica primaria como de secundaria) no 
hacen alusión al proceso o las instituciones que administran justicia; en su cotidianidad no viven la justicia desde allí sino desde valores positivos que se materializan en las relaciones que entablan con otros en la escuela. La discordancia pareciera estar en el núcleo central, en el cual se contraponen los valores positivos con elementos negativos que se refieren a la violencia y la venganza.

Los resultados obtenidos en colegios privados difieren en algunos aspectos de aquellos que provienen de estudiantes de colegios oficiales. Como se evidencia en la tabla 4, las palabras que se encuentran dentro del núcleo central de la RS de la justicia en los infantes de básica primaria de colegio privado son variadas. Inicialmente, se encuentran palabras asociadas a valores e ideales como lo son libertad, paz, respeto $\mathrm{y}$ derechos. No obstante, pueden verse palabras tales como venganza relacionadas con un tipo de justicia de carácter retributivo (Carlsmith \& Darley, 2008; Okimoto et al., 2012)-y otras como democracia, politica y norma las cuales aluden a procesos formales relacionados con la justicia. Junto con estas, también se encuentra la palabra corrupción, la cual pareciera ser el quiebre entre los valores ideales que se incluyen en este núcleo y los procesos mediante los cuales se imparte justicia. La corrupción ya ha sido propuesta como parte de los modelos mentales que enmarcan el razonamiento de universitarios colombianos cuando analizan la producción agro-minera en Colombia (GutiérrezRomero, 2018).

La confluencia de conceptos tan variados dentro de este núcleo central puede explicarse a la edad de los participantes ya que en las edades correspondientes a la básica primaria no suele haber una estructura sólida en el núcleo central (Flores \& Jiménez, 2015) lo cual es similar a lo encontrado en el análisis estructural de la RS de los infantes de primaria en colegio oficial. En la primera periferia de la RS de los infantes de básica primaria de colegio privado (tabla 4), pueden verse palabras que parecieran referirse a posibles alternativas frente a la problemática de la justicia (anticorrupción, solución) y diferentes figuras o mecanismos que lleven a cabo dichas soluciones (policía, cárcel, juez y abogado). Llama la atención que los infantes de colegio privado 
se refieren con mayor frecuencia a soluciones y alternativas frente al concepto de justicia en comparación con los niños y niñas de colegio oficial. Además, los infantes de colegio privado hacen mayor alusión a conceptos relacionados con las instituciones o procesos de administración de justicia que aquellos de colegio oficial sugiriendo así, que tienen un mayor acercamiento a estos procesos y por ende un mayor conocimiento de los mismos.

Un último factor que llama la atención es el hecho de que en el análisis estructural de la RS de los infantes de colegio privado no aparece ninguna palabra que haga alusión a las armas mientras que en el análisis de los infantes de colegio oficial sí aparecen este tipo de palabras. Esto puede deberse a que los colegios públicos en Colombia suelen estar ubicados en zonas donde la vulnerabilidad y la violencia son factores concurrentes con los que sus habitantes, incluidos los niños, tienen que enfrentarse de forma cotidiana.

Finalmente, en el núcleo central de la RS sobre la justicia del grupo de estudiantes de secundaria de colegios privados, pueden verse palabras relacionadas con valores e ideales tales como igualdad, equidad, honestidad y libertad. Además de esto, puede verse que el concepto de justicia está ligado al poder (palabra presente en el núcleo central de la RS). Pareciera que quien imparte la justicia es quien tiene el poder. Allí también se encuentra la palabra corrupción la cual podría estar ligada al ejercicio de poder que se mencionó anteriormente y podría representar el factor que distorsiona los valores ideales de la justicia ya mencionados. Así, puede verse que el núcleo central de la RS de la justicia de estudiantes de secundaria de colegios privados reúne elementos y valores ideales que son contaminados por un ejercicio de poder que da como resultado la corrupción.

Esto difiere de lo encontrado con respecto a los adolescentes de colegios oficiales, ya que en ellos lo que irrumpe el ideal de justicia es la violencia y la venganza, más no la corrupción. Cabría preguntarse si el origen de esta diferencia está ligado al contexto socioeconómico en el que han crecido esto jóvenes: unos dentro de entornos permeados por los conflictos violentos y otros dentro de contextos con una mayor 
cercanía al poder económico y social y a su vez, a la corrupción. Esto concordaría con lo planteado por Fasanelli et al., (2014) quienes plantean que las representaciones de la noción de justicia en adolescentes se ven influenciadas por el estrato socio-económico.

En cuanto a la primera periferia de la RS que los adolescentes de colegio privado tienen de la justicia, es decir, la forma como la viven en su cotidianidad, se evidencian tres grupos de palabras. El primero hace referencia a ideales y valores, pues incluye las palabras paz, verdad, ética, moral, bienestar, respeto. El segundo grupo de palabras está ligado a una visión más procesual de la justicia desde los entes formales que la administran, lo cual puede verse en las palabras politica, juez, corte, juicio y abogado. Por último, están las palabras relacionadas con una visión retributiva de la justicia (Carlsmith \& Darley, 2008; GutiérrezRomero, et al., 2020; Okimoto, et al., 2012) como lo son cárcel y castigo. Se evidencia que los pertenecientes a este grupo se refieren no solo a qué es la justicia desde lo ideal, sino también al cómo se ejecuta. Esto en contraposición a lo encontrado en los colegios oficiales, en donde no se hace referencia a los procesos de ejecución de la justicia.

Desde una perspectiva del desarrollo, se puede discutir diversos puntos cruciales para entender la relación que establecen en la infancia con el concepto de justicia. La psicología del desarrollo ha mostrado que los niños desarrollan un cierto sentido de la justicia desde un principio: los bebés de diez meses esperan que los recursos se asignen por igual (Meristo, \& Surian, 2013), los niños de 4 y 6 años muestran una marcada aversión a la inequidad (Qiu et al., 2017) y niños después de los 9 ańos de edad comienzan a moderar su objetivismo con juicios meta-éticos culturalmente relativos sobre la justicia (Schmidt, 2017). Este estudio es pionero en encontrar que las RS, en este caso sobre la justicia, tienen una estructura definida aun en épocas tempranas de la educación formal. Desde segundo grado estas tienen referentes específicos con los cuales explicar y predecir la realidad que se percibe; también se halló que el referente sobre el cual la justicia toma forma cambia con la edad pues se pasa de una realidad perceptible en lo coti- 
diano como los elementos físicos con los que se ejerce violencia a los ideales que caracterizan la justicia.

La variable estrato socioeconómico modifica también la forma de comprender los fenómenos asociados a la justicia pues las realidades que estructuran las RS pasan de la violencia armada y sus actores a fenómenos menos tangibles (al menos directamente) como la corrupción. Se ha propuesto que el conflicto social y sus diferentes niveles de gravedad se manifiestan en diversas problemáticas que son perceptibles por niños y niñas en el tiempo (Martínez, \& Zuluaga, 2019); pareciese entonces que la importancia atribuida a diferentes tipos de conflictos cambia en el ciclo vital y que la justicia adquiere su importancia al poder regularlas para beneficio de la sociedad.

Chan (2008) ha propuesto que los agentes morales hacen lo que juzgan como justo; los juicios morales son entonces juicios evaluativos, es decir, conciernen a los puntos de vista de los agentes morales sobre el valor de hacer algo en lugar de otra cosa. Los resultados de esta investigación dan cuenta que los infantes, en tanto agentes morales, proyectan sus razonamientos morales sobre actores y fenómenos sociales específicos y que sobre ellos se privilegia el deseo de accionar de la justicia. La apreciación del valor tiene fuerza normativa o guía de acción para el agente moral; para autores como Janoff-Bulman y Carnes (2013) y Hofmann et al., (2014), el conocimiento conceptual en el juicio moral concierne a quién o qué es lastimado y cómo está siendo perjudicado; siendo consistentes con lo propuesto se esperaría que estos infantes proyecten sus acciones sobre los fenómenos que resultan susceptibles de ser intervenidos por la justicia. Dado que la comprensión de la justicia y el perdón son piezas centrales en procesos como la confianza y la reconciliación (Mullet et al., 2016) la justicia se perfila en nińos y nińas como una forma para alcanzar cierto equilibrio en sus vivencias cotidianas y así lograr cierta confianza en la sociedad.

La manera como los infantes y adolescentes de instituciones privadas se aproximan al concepto aquí estudiado es cercana a la propuesta de Amartya Sen $(1977 ; 2010)$ con respecto al mejoramiento de la injusticia y la inclusión de fórmulas para superar la injusticia (Campos, 
2011; Colmenarejo, 2013; 2016; Nussbaum, 2007). En general, puede verse que la RS de la justicia de los estudiantes de colegios privados (tanto de primaria como de secundaria) incluye más soluciones frente a la problemática que implica este concepto y una mayor cantidad de mecanismos para ejercer justicia, que aquella de los estudiantes pertenecientes a colegios oficiales. Es decir, los estudiantes de colegios privados parecen estar alineados con la intención de Sen de "esclarecer cómo podemos plantearnos la cuestión del mejoramiento de la injusticia, en lugar de ofrecer respuestas a las preguntas sobre la naturaleza de la justicia perfecta” (Sen, 2011). Ese es entonces el centro de la discusión de la teoría de la justicia propuesta por Sen: establecer cuáles son las injusticias reparables y tratar de eliminarlas. Las RS de los estudiantes de colegios privados muestran que estos suelen plantearse más alternativas frente a las injusticas de las que son testigos, que los estudiantes de colegios públicos. Esto se ve reflejado en la presencia de palabras como "solución", para el caso de los niños y niñas de básica primaria, u otras como "juez" o "abogado", las cuales están presentes tanto en niños y nińas, como en adolescentes de colegios privados, representando un medio para eliminar o al menos disminuir ciertas injusticias. Esto puede deberse a que los estudiantes de colegios privados pertenecen a un círculo social que les permite tener más acceso a profesiones y mecanismos relacionados con la atención de la injustica y por ende no sienten tanta impotencia frente a su existencia, como pueden sentir los estudiantes de colegios públicos.

\section{Conclusiones}

Los resultados anteriormente discutidos muestran que existen diferencias en la estructura de la representación de la justicia en relación con el estrato socioeconómico. Se evidencia que tanto los estudiantes de colegio privado como los de colegio público, tienen un ideal de justicia que se contrapone con elementos negativos los cuales, en el caso de los colegios oficiales, están ligados a la violencia y la venganza mientras que 
en los colegios privados están relacionados con la corrupción. Además, se identificó que la representación de la justicia de los estudiantes de colegios privados incluye más soluciones frente a la problemática que implica este concepto y una mayor cantidad de mecanismos para ejercerlo, que aquella de los estudiantes pertenecientes a colegios oficiales. El origen de esta diferencia puede ligado al contexto socioeconómico en el que han crecido esto jóvenes: unos en entornos permeados por los conflictos violentos y otros en contextos con una mayor cercanía al poder económico y social y a su vez, a la corrupción.

Esta investigación evidencia que, como se mencionó anteriormente, el contexto en el que viven los niños, niñas y adolescentes colombianos, ha influenciado sus enmarques emocionales y cognitivos relacionados con temas como la venganza y la consolidación de prácticas culturales violentas (Castrillón-Guerrero et al., 2018; LópezLópez, 2016a; López-López, 2016b). Además, dicho contexto ha influido en procesos sociopsicológicos asociados a la justicia y en la forma como esta población representa y le da sentido a este concepto. Esto abre futuras discusiones y líneas de investigación en torno a la causalidad entre las particularidades del contexto socioeconómico y la construcción de representaciones sociales sobre justicia. En el caso de los infantes y adolescentes colombianos, ahondar en estos temas permitirá comprender la forma como construyen su realidad social y se comportan en ella, e intervenir en la estructuración de los futuros ciudadanos de Colombia.

\section{Referencias}

Abric, J. C. (2001a). Prácticas sociales y representaciones. México: Coyoacán

Abric, J.C. (2001b). L'approche structurale des représentations sociales: développements récents. Psychologie et Société, 4(2), 81-104. Disponible en https:/www.academia.edu/download/34799735/ 
Abric_-_Unknown_-_Lapproche_structurale_des_representations_sociales_developpements_recents.pdf

Barreiro, A., \& Castorina, A. (2015). La creencia en un mundo justo como trasfondo ideológico de la representación social de la justicia. Revista Colombiana de Psicologia, 24(2), 331-345. https:// dx.doi.org/10.15446/rcp.v24n2.44294

Barreiro, A., Gaudio, G., Mayor, J., Santellán-Fernández, R., Sarti, D. \& Sarti, M. (2014). Justice as social representation: Diffusion and differential positioning. Revista de Psicología Social, 29(2), 319-341. https://doi.org/10.1080/02134748.2014.918821

Betancourt, C. A. G. (2019). El lugar de la psicología en los procesos de justicia restaurativa. Poiésis, 37, 206-211. https://doi. org/10.21501/16920945.3344

Bolívar, A. (2012). Justicia social y equidad escolar. Una revisión actual. Revista Internacional de Educación para la Justicia Social, 1(1), 9-45. Disponible en http://repositorio.minedu.gob.pe/ handle/123456789/1589

Borja, H., Barreto, I., Alzate, M., Sabucedo, J. M., \& López, W. L. (2009). Creencias sobre el adversario, violencia política y procesos de paz. Psicothema, 21(4), 622-627. Disponible en https://www.unioviedo.net/reunido/index.php/PST/article/ view/8831/8695

Bruno, D., \& Barreiro, A. (2014). La política como representación social. Psicología Politica, 48, 69-80. Disponible en https://www. uv.es/garzon/psicologia\%20politica/N48-4.pdf

Campos, H. (2011). La justicia posible: comentario sobre The idea of justice de Amartya Sen. Revista de Economía Institucional, 13(24), 459-464. Disponible en https://dialnet.unirioja.es/descarga/articulo/6281672.pdf

Carlsmith, K.M., \& Darley, J.M. (2008). Psychological aspects of retributive justice. Advances in Experimental Social Psychology, 40, 193-236. https://doi.org/10.1016/S0065-2601(07)00004-4 Castrillón-Guerrero, L., Riveros, V., Knudsen M. L., López-López, W., Correa-Chica, A. \& Castañeda, J.G. (2018). Comprensiones de 
perdón, reconciliación y justicia en víctimas de desplazamiento forzado en Colombia. Revista de Estudios Sociales 63: 84-98. https://dx.doi.org/10.7440/res63.2018.07

Chan, D. K. (2008). Moral psychology today: Essays on values, rational choice, and the will (Vol. 110). Springer Science \& Business Media.

Colmenarejo, R. (2013). La idea de la justicia de Amartya Sen, un tratado sobre la injusticia. Revista de Fomento Social, 68, 43-58. Disponible en https:/www.revistadefomentosocial.es/rfs/ article/view/1725

Colmenarejo, R. (2016). Enfoque de capacidades y sostenibilidad Aportaciones de Amartya Sen y Martha Nussbaum. Ideas y valores, 65(160), 121-149. Disponible en https://revistas.unal. edu.co/index.php/idval/article/view/43084

Dandurand, Y., \& Griffiths, C.T. (2006). Handbook on Restorative Justice Programmes. United Nations Publications

Denegri, M., Chávez, D., Silva, F., \& del Valle, C. (2015). Representaciones sociales en torno al "conflicto" estado chileno-pueblo mapuche en jóvenes universitarios chilenos: Un estudio con redes semánticas naturales. Investigación \& Desarrollo, 23(2), 256-277. http://dx.doi.org/10.4482/indes.23.2.7065

Echeverry, Y., \& Jaramillo, J. (2006). El concepto de justicia en John Rawls. Revista Cientifica Guillermo de Ockham, 4(2), 27-52. Disponible en https://dialnet.unirioja.es/descarga/articulo/2877302.pdf

Fasanelli, R., Galli, I., \& Liguori, A. (2014). Aveugle ou tout simplement myope? La représentation sociale de la justice chez des étudiants provenant de contextes socio-économiques différents. Les Cahiers Internationaux de Psychologie Sociale, 103, 469-495. https://doi.org/10.3917/cips.103.0469

Flores, G., \& Jiménez, M.S. (2015). Las representaciones sociales sobre derechos humanos en niños de primaria. Revista Electrónica de Investigación Educativa, 17(3), 116-131. Disponible en //www. 
scielo.org.mx/scielo.php?script=sci_arttext\&pid=S1607-40412 015000300009\&lng=es\&tlng=es

Forsé, M., Richardot, S., Frénod, A., Guibet-Lafaye, C., \& Parodi, M. (2016).

Gollwitzer, M., \& J.W. van Prooijen (2016): Psychology of justice. In C. Sabbagh \& M. Schmitt (Eds.), Handbook of Social Justice Theory and Research (pp. 61-82). Springer New York, NY.

Graybill, E., Baker, C. N., Cloth, A. H., Fisher, S., \& Nastasi, B.

K. (2018). An analysis of social justice research in school psychology. International Journal of School \& Educational Psychology, 6(2), 77-89. https://doi.org/10.1080/21683603.2017.130 2850

Grocke, P., Rossano, F., \& Tomasello, M. (2015). Procedural justice in children: Preschoolers accept unequal resource distributions if the procedure provides equal opportunities. Journal of Experimental Child Psychology, 140, 197-210.https://doi. org/10.1016/j.jecp.2015.07.008

Gutiérrez-Romero, M.F. (2018). Socioscientific argumentation and model-based reasoning: A study on mining exploitation in Colombia. Universitas Psychologica, 17(5), 1-12. https://doi. org/10.11144/Javeriana.upsy17-5.samb

Gutiérrez-Romero, M.F., López-López, W., \& Silva, L.M. (2020). Preferencia hacia la justicia restaurativa y retributiva y su relación con el nivel de competencia moral. Suma Psicológica, 27(2), 107-115. https://doi.org/10.14349/sumapsi.2020.v27.n2.5

Hofmann, W., Wisneski, D. C., Brandt, M. J., \& Skitka, L. J. (2014). Morality in everyday life. Science, 345(6202), 1340-1343. https:// doi.org/10.1126/science.1251560

House, B., Henrich, J., Sarnecka, B., \& Silk, J. B. (2013). The development of contingent reciprocity in children. Evolution and Human Behavior, 34(2), 86-93. https://doi.org/10.1016/j. evolhumbehav.2012.10.001

Instituto Nacional de Medicina Legal y Ciencias Forenses. (2019). Forensis 2018. Datos para la vida. Disponible en 
http://www.medicinalegal.gov.co/documents/20143/386932/ Forensis+2018.pdf/be4816a4-3da3-1ff0-2779-e7b5e3962d60 Janoff-Bulman, R., \& Carnes, N. C. (2013). Surveying the moral landscape: Moral motives and group-based moralities. Personality and Social Psychology Review, 17, 219-236. https:// doi.org/10.1177/1088868313480274

Jodelet, D. (1986): La representación social: Fenómenos, concepto y teoría. En Serge Moscovici, Pensamiento y vida social. Psicología social y problemas sociales. Psicología social II. Barcelona: Paidós.

Jodelet, D. (2006). El otro, su construcción, su conocimiento. En Abundiz Valencia (Coord.) Representaciones sociales. Alteridad, epistemología y movimientos sociales. México: Universidad de Guadalajara.

Kenward, B., Hellmer, K., Winter, L.S., \& Eriksson, M. (2015). Four-year-olds' strategic allocation of resources: Attempts to elicit reciprocation correlate negatively with spontaneous helping. Cognition, 136, 1-8. https://doi.org/10.1016/j. cognition.2014.11.035

Kohlberg, L. (1969). Stage and sequence; The cognitive-developmental approach to socialization.

Kohlberg, L. (1984). Essays on moral development: The psychology of moral development (Vol. 2). New York: Harper \& Row.

Lahlou, S., \& Abric, J. C. (2011). What are the "elements" of a representation?. Papers on Social Representations, 20, 20-1. Disponible en http://eprints.lse.ac.uk/44355/

López-López, W. (2016a). La reconstrucción psicológica y social: una prioridad de corto, mediano y largo plazo para el futuro de la paz en Colombia. En La paz el derecho de la democracia. Elementos de análisis frente a los desafios del legislador en la implementación del acuerdo final de paz en Colombia, editado por Giovanny Niño, Julián Escobar y Lucio Muñoz, 149-157. Popayán: Centro de Investigaciones de Altos Estudios Legislativos - Senado de la República de Colombia - Universidad del Cauca. 
López-López, W. (2016b). El perdón como condición para la construcción de una paz sostenible. El Espectador, enero 15. http://www. elespectador.com/n oticias/paz/ el-perdon-condicion-construccion-de-una-paz-sostenible-articulo-610768

López-López, W., Rincón-Unigarro, C., Gutiérrez-Romero, M.F., \& Rodríguez-Cely, L. (2019). Psicología de la justicia para la construcción de paz en Colombia: Una apuesta desde la psicología política. En Álvaro Díaz Gómez \& Omar Alejandro Bravo, Psicología Politica y Procesos para la Paz en Colombia (pp. 121-150), Cali, Colombia: Editorial Universidad ICESI y ASCOFAPSI.

Martínez, A., \& Zuluaga, D. A. P. (2019). Justicia y conflicto infantil. Via Inveniendi Et Iudicandi, 14(1), 191-206. Disponible en https://dialnet.unirioja.es/servlet/articulo?.codigo=6860273

Meristo, M., \& Surian, L. (2013). Do infants detect indirect reciprocity?. Cognition, 129, 102-113. https://doi.org/10.1016/j. cognition.2013.06.006

Migliore, J. (2011). Amartya Sen: La idea de la justicia. Cultura Económica, 29(81-82), 13-26. Disponible en:http://bibliotecadigital. uca.edu.ar/repositorio/revistas/amartya-sen-idea-justicia.pdf

Molina, J. (2017). Tutorial para el análisis de textos con el software Iramuteq. Disponible en https:/www.researchgate.net/ publication/315696508_Tutorial_para_el_analisis_de_textos_ con_el_software_IRAMUTEQ

Montero, I., \& León, O. G. (2005). Sistema de clasificación del método en los informes de investigación en Psicología. International Journal of Clinical and Health Psychology, 5(1), 115-127. Disponible en https://www.redalyc.org/articulo.oa?id=33701007

Moscovici, S. (1961). La psychanalyse, son image et son public: Etude sur la représentation sociale de la psychanalyse. Presses Universitaires de France.

Mullet, E., López- López, W., Kpanake, L., Mukashema, I., Armange, R., Kamble, S.,... Neto, F. (2016). Functional measurement in the field of ethics in politics. Universitas Psychologica, 15(3), 292-344. http://dx.doi.org/10.11144/Javeriana.upsy15-3.fmf 
Nussbaum, M. C. (2007). Las fronteras de la justicia. Barcelona: Ediciones Paidós Ibérica, S.A.

Observatorio del Bienestar de la Niñezy el Instituto Colombiano de Bienestar Familiar. (2018). Ingresos de niños, niñasy adolescentes a PARD por motivo de maltrato. Recuperado de https://www.icbf.gov.co/ programas-y-estrategias/observatorio-del-bienestar-de-la-ninez/ ingresos-de-ninos-ninas-y

Okimoto, T.G., Wenzel, M., \& Feather, N.T. (2012). Retribution and restoration as general orientations toward justice. European Journal of Personality, 26, 255-275. https://doi.org/10.1002/ per.831

Orjuela, A. (2018). Representaciones sociales de la justicia en estudiantes universitarios de Colombia. (Tesis de pregrado). Pontificia Universidad Javeriana, Bogotá.

Paulus, M. (2015). Children's inequity aversion depends on culture: A cross-cultural comparison. Journal of Experimental Child Psychology, 132, 240-246. http://dx.doi.org/10.1016/j. jecp.2014.12.007

Piaget, J. (1932). El juicio moral del niño. Madrid, Francisco Beltrán Editor.

Qiu X., Yu J., Li T., Cheng N., \& Zhu L. (2017). Children's inequity aversion in procedural justice context: A comparison of advantageous and disadvantageous inequity. Frontiers in Psychology, 8, 1855. https://doi.org/10.3389/fpsyg.2017.01855

Ramírez, D. A., de la Rosa, E. A., \& Valencia, N. M. (2018). Justicia retributiva y restaurativa: Análisis comparado a través de estudios de caso en el Valle del Cauca. Revista Iberoamericana de Psicología, 11(1), 55-64. Disponible en https://dialnet.unirioja. es/servlet/articulo?codigo $=6598580$

Rawls, J. (1997). Teoría de la justicia. México: Fondo de Cultura Económica.

Rest, J. R. (1973). The hierarchical nature of moral judgment. Journal of Personality, 41, 86-109. https://doi. org/10.1111/j.1467-6494.1973.tb00662.x 
Rest, J. R. (1979). Development in Judging Moral Issues. Minneapolis: MN: The University of Minnesota Press.

Rest, J. R., Narvaez, D., Bebeau, M. J., \& Thoma, S. J. (1999). Postconventional moral thinking: A neo-Kohlbergian approach. Mahwah, NJ: Erlbaum.

Rochat, P., Dias, M. D., Liping, G., Broesch, T., Passos-Ferreira, C., Winning, A., \& Berg, B. (2009). Fairness in distributive justice by 3 -and 5 -year-olds across seven cultures. Journal of Cross-Cultural Psychology, 40(3), 416-442. https://doi. org/10.1177/0022022109332844

Romero, J. J., \& Ramírez, O. O. (2014). L'injustice comme expression de la pensée sociale au Mexique. Les Cahiers Internationaux de Psychologie Sociale, 103, 497-516. https://doi.org/10.3917/ cips.103.0497

Sabbagh, C., \& Schmitt, M. (2016). Past, present, and future of social justice theory and research. In C. Sabbagh \& M. Schmitt (Eds.), Handbook of Social Justice Theory and Research (pp. 1-11). Springer, New York, NY

Sánchez, G. A. A., Ossa, S. Y. D., \& Méndez, F. F. (2019). Las nociones de justicia que construyen las niñas en pleno siglo XXI. Via Inveniendi et Iudicandi, 14(1), 67-89. Disponible en https://dialnet. unirioja.es/servlet/articulo?codigo $=6860275$

Sarmiento-López, J. C., \& Yáñez-Canal, J. (2019). Discusiones políticas y morales referentes a la justicia y su aporte a la psicología del desarrollo moral. Revista Colombiana de Psicología, 28, 165-179. https://doi.org/10.15446/rcp.v28n1.70636

Schmidt, M. F., Svetlova, M., Johe, J., \& Tomasello, M. (2016). Children's developing understanding of legitimate reasons for allocating resources unequally. Cognitive Development, 37, 42-52. https://doi.org/10.1037/a0040069.

Schmidt, M.F., Gonzalez-Cabrera, I., \& Tomasello, M. (2017). Children's developing metaethical judgments. Journal of Experimental Child Psychology, 164, 163-177. http://dx.doi. org/10.1016/j.jecp.2017.07.008 
Sen, A. (1997). Bienestar, justicia y mercado. Ediciones Paidós Ibérica. Sen, A. (2010). La idea de la justicia. Bogotá: Distribuidora y Editora Aguilar, Altea, Taurus, Alfaguara S.A.

Shinn, M. (2015). Community psychology and the capabilities approach. American Journal of Community Psychology, 55(3-4), 243-252. https://doi.org/10.1007/s10464-015-9713-3

Wenzel, M., \& Okimoto, T.G. (2014). On the relationship between justice and forgiveness: Are all forms of justice made equal?. British Journal of Social Psychology, 53(3), 463-483. https://doi. org/10.1111/bjso. 12040

Wittig, M., Jensen, K., \& Tomasello, M. (2013). Five-year-olds understand fair as equal in a mini-ultimatum game. Journal of Experimental Child Psychology, 116(2), 324-337. https://doi. org/10.1016/j.jecp.2013.06.004

Recibido: 11 de noviembre, 2019

Revisado: 28 de julio, 2020

Aceptado: 4 de marzo, 2021 